\title{
Article
}

\section{Electronic States of Silicene Allotropes on Ag(111)}

Polina M. Sheverdyaeva, Sanjoy Kr. Mahatha, Paolo Moras, Luca

Petaccia, Guido Fratesi, Giovanni Onida, and Carlo Carbone

ACS Nano, Just Accepted Manuscript • DOI: 10.1021/acsnano.6b07593 • Publication Date (Web): 29 Dec 2016

Downloaded from http://pubs.acs.org on January 3, 2017

\section{Just Accepted}

"Just Accepted" manuscripts have been peer-reviewed and accepted for publication. They are posted online prior to technical editing, formatting for publication and author proofing. The American Chemical Society provides "Just Accepted" as a free service to the research community to expedite the dissemination of scientific material as soon as possible after acceptance. "Just Accepted" manuscripts appear in full in PDF format accompanied by an HTML abstract. "Just Accepted" manuscripts have been fully peer reviewed, but should not be considered the official version of record. They are accessible to all readers and citable by the Digital Object Identifier (DOI®). "Just Accepted" is an optional service offered to authors. Therefore, the "Just Accepted" Web site may not include all articles that will be published in the journal. After a manuscript is technically edited and formatted, it will be removed from the "Just Accepted" Web site and published as an ASAP article. Note that technical editing may introduce minor changes to the manuscript text and/or graphics which could affect content, and all legal disclaimers and ethical guidelines that apply to the journal pertain. ACS cannot be held responsible for errors or consequences arising from the use of information contained in these "Just Accepted" manuscripts. 
(4×4)

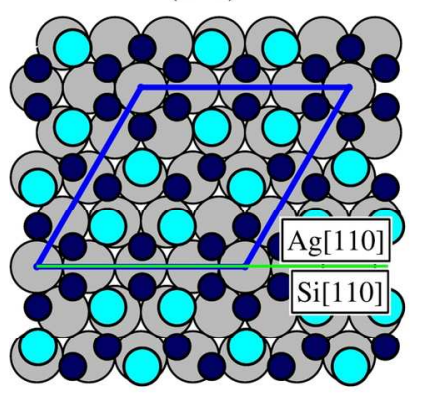

a

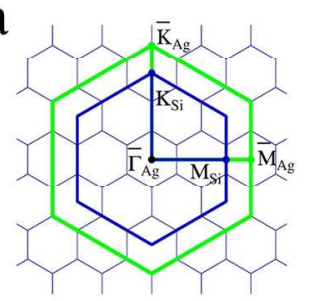

$(\sqrt{ } 13 \times \sqrt{ } 13) R 13.9^{\circ}$

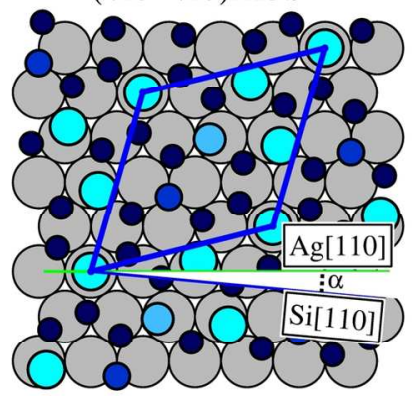

b

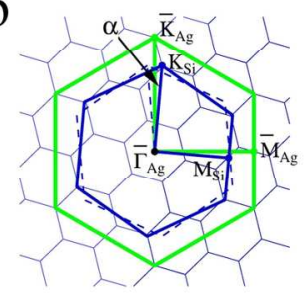

$(2 \sqrt{ } 3 \times 2 \sqrt{ } 3) \mathrm{R} 30^{\circ}$

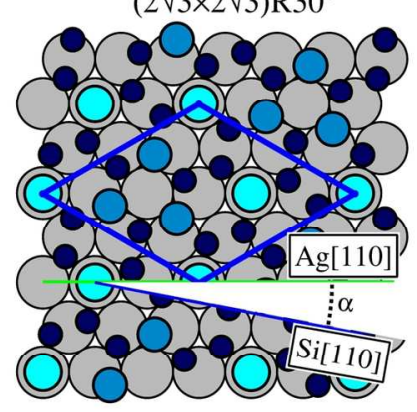

c

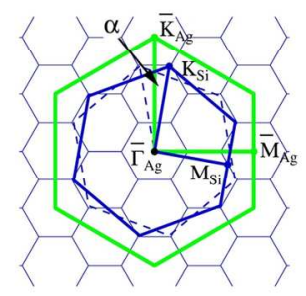

Figure 1. Top row: structural model of the silicene allotropes on $\mathrm{Ag}(111)$ (derived from ref 18 ). Grey circles represent $\mathrm{Ag}$ atoms. Blue circles represent $\mathrm{Si}$ atoms located at different height (circle size and color brightness increase with increasing the distance from the Ag substrate). Blue rhombi define the unit cells of the silicene superstructures. The angle a between the $\mathrm{Si}[110]$ and $\mathrm{Ag}$ [110] directions is characteristic of each phase $(a)(4 \times 4), a=0^{\circ}$; (b) $(\sqrt{ } 13 \times \sqrt{ } 13) R 13.9^{\circ}, a=5.2^{\circ} ;$ (c) $(2 \sqrt{ } 3 \times 2 \sqrt{ } 3) R 30^{\circ}, a=10^{\circ}$. Bottom row: reciprocal space of the silicene allotropes on $\mathrm{Ag}(111)$. Thin blue hexagons indicate the SBZ of the silicene reconstruction. Thick blue and green hexagons represent the $(1 \times 1) \mathrm{SBZ}$ of silicene and $\mathrm{Ag}(111)$, respectively. Dashed hexagons in (b) and (c) are used to highlight the presence of a second rotational domain.

$150 \times 88 \mathrm{~mm}(300 \times 300 \mathrm{DPI})$ 
Figure 2. (a) LEED pattern at $31 \mathrm{eV}$ of the $(4 \times 4) /(\sqrt{ } 13 \times \sqrt{ } 13) \mathrm{R} 13.9^{\circ}$ system. Some first-order diffraction spots of $\mathrm{Ag}$ and silicene are indicated by arrows. The oval area encloses first-order diffraction spots of silicene back-scattered from the second SBZ of Ag. The blurred spots in the squared area are associated to the low-ordered $(\sqrt{ } 13 \times \sqrt{ } 13) R 13.9^{\circ} \mathrm{b}$ phase. (b) Schematic diagram of the LEED pattern of panel (a) that shows the origin of the back-scattered silicene spots (blue dots) by dashed circular lines. (c) LEED pattern at $31 \mathrm{eV}$ of the $(2 \sqrt{ } 3 \times 2 \sqrt{ } 3) \mathrm{R} 30^{\circ}$ system. (d) Same as panel (b) for the $(2 \sqrt{ } 3 \times 2 \sqrt{ } 3) \mathrm{R} 30^{\circ}$ system.

$137 \times 145 \mathrm{~mm}(300 \times 300 \mathrm{DPI})$ 

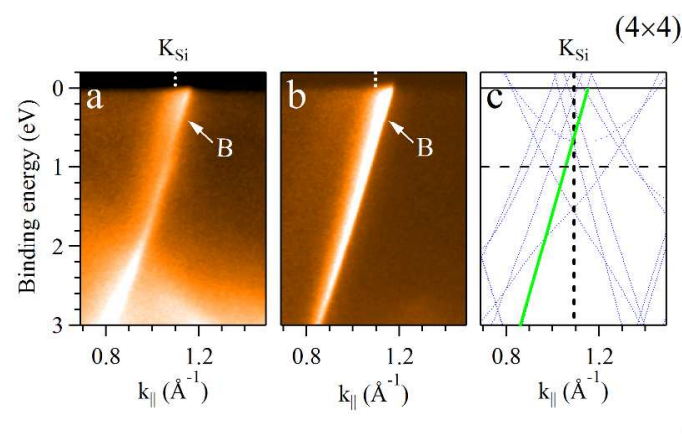

$(4 \times 4) /(\sqrt{ } 13 \times \sqrt{ } 13) R 13.9^{\circ}$
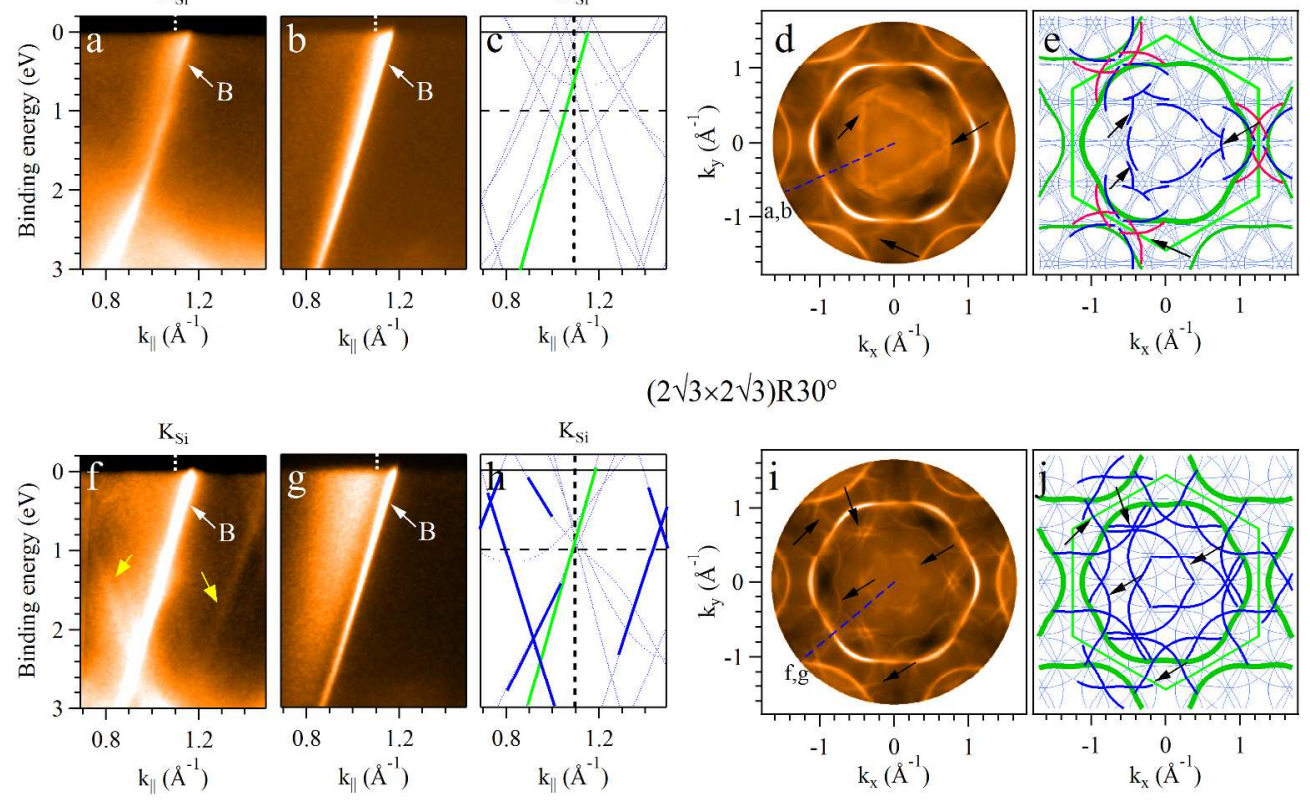

$(2 \sqrt{ } 3 \times 2 \sqrt{ } 3) \mathrm{R} 30^{\circ}$

Figure 3. Top row: ARPES analysis ( $31 \mathrm{eV}$ photon energy) and simulations for the $(4 \times 4) /(\sqrt{ } 13 \times \sqrt{ } 13) \mathrm{R} 13.9^{\circ}$ system. (a) ARPES data measured along the line indicated in panel (d) near the Ksi point of the $(\sqrt{13} \times \sqrt{ } 13) R 13.9^{\circ}$ phase. The white arrow indicates the B band. (b) Same as panel (a) for the clean $\mathrm{Ag}(111)$. (c) Simulation of the band dispersion of panel (a), where thick green and thin blue lines represent the original B band and its umklapp replicas according to the $(4 \times 4)$ periodicity. (d) Constant energy cut of the ARPES data at $1 \mathrm{eV}$ below EF. (e) Simulation of the band pattern of panel (d). Thick green and thin blue lines represent the energy contour of the original B band and its umklapp replicas according to the $(4 \times 4)$ periodicity. Thick blue lines highlight the umklapp bands observed in the experiment. Similarly, thick red

lines mark the features observed in the experiments that derive from umklapp replicas of the $B$ band repeated according to the $(\sqrt{ } 13 \times \sqrt{ } 13) R 13.9^{\circ}$ periodicity (see text for more details). Black arrows facilitate the comparison between the ARPES pattern and the simulation. Bottom row: same as the top row for the $(2 \sqrt{ } 3 \times 2 \sqrt{ } 3) R 30^{\circ}$ system. Thick blue lines in panel $(h)$ indicate some of the umklapp replicas of the $B$ band observed in panel $(f)$. 
Figure 4. Top row: ARPES data (31 eV photon energy) for the $(4 \times 4) /(\sqrt{ } 13 \times \sqrt{ } 13) \mathrm{R} 13.9^{\circ}$ system measured in the proximity of the $M \_A g$ point along the (a) $\Gamma \_A g-M \_A g$ and (b) $K \_A g-M \_A g-K \_A g$ directions. Line scans are shown in panel (e) with reference to the Ag SBZ. Yellow arrows indicate umklapp replicas of the B band. Bottom row: same as the top row for the $(2 \sqrt{ } 3 \times 2 \sqrt{ } 3) \mathrm{R} 30^{\circ}$ system.

$$
117 \times 243 \mathrm{~mm}(300 \times 300 \mathrm{DPI})
$$



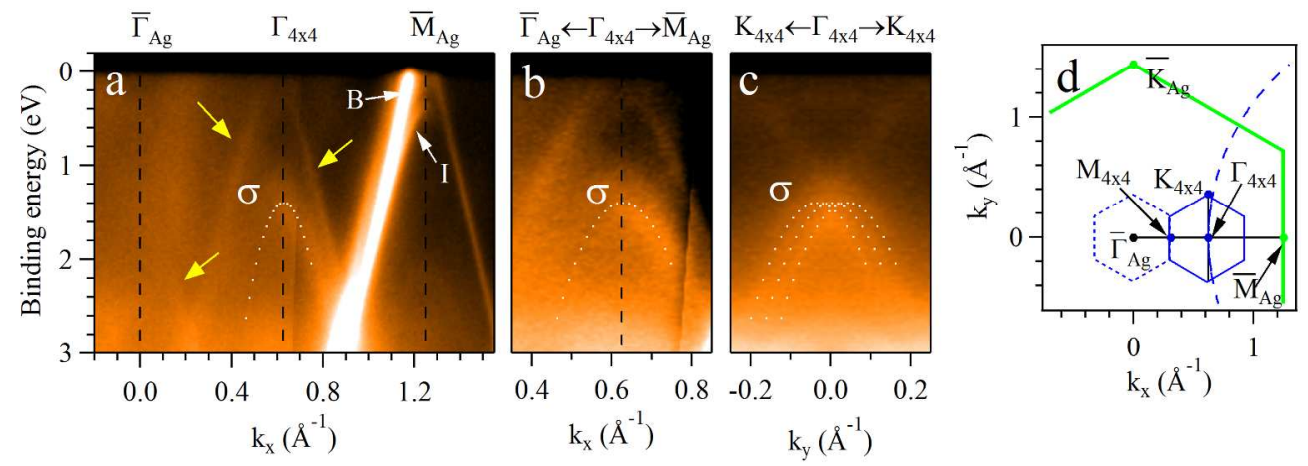

$(2 \sqrt{3} \times 2 \sqrt{ } 3) \mathrm{R} 30^{\circ}$
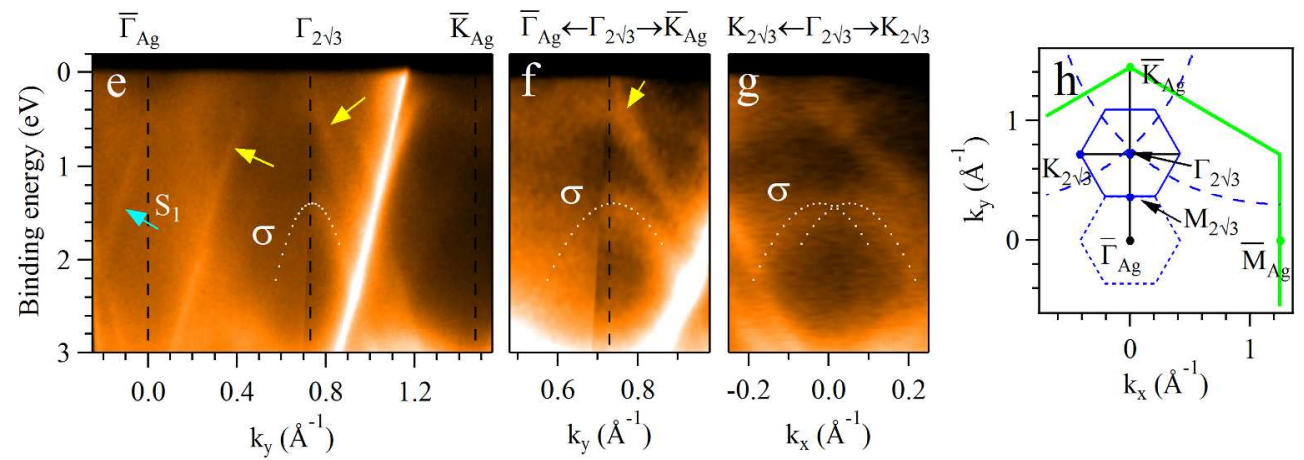

Figure 5. Top row: $\sigma$ bands of the $(4 \times 4) /(\sqrt{ } 13 \times \sqrt{ } 13) R 13.9^{\circ}$ system. ARPES scans along the $(a, b) \Gamma \_A g-$ M_Ag and (c) K_4 $4 \times 4-\Gamma \_4 \times 4-K_{-} 4 \times 4$ directions (photon energy $31 \mathrm{eV}$ ). Dashed lines highlight the $\sigma$ band dispersions. Yellow arrows in panel (a) indicate umklapp replicas of the B band. (d) Location of the symmetry points and SBZ (blue hexagons) of the $(4 \times 4)$ superstructure with reference to the $\mathrm{Ag}(111) \mathrm{SBZ}$ (green hexagon). Bottom row: $\sigma$ bands of the $(2 \sqrt{3} \times 2 \sqrt{3})$ R3 $30^{\circ}$ system. ARPES scans along the $(e, f) \Gamma \_A g-$ $\mathrm{K} \_\mathrm{Ag}$ and $(\mathrm{g}) \mathrm{K} \_2 \sqrt{3}-\Gamma \_2 \sqrt{ } 3-\mathrm{K} \_2 \sqrt{3}$ directions (photon energy $31 \mathrm{eV}$ ). Dashed lines highlight the $\sigma$ band dispersions. Arrows in panel (e) indicate umklapp replicas of the B band (yellow) as well as the S1 state (cyan). (f) Location of the symmetry points and SBZ (blue hexagons) of the $(2 \sqrt{ } 3 \times 2 \sqrt{ } 3) \mathrm{R} 30^{\circ}$ superstructure with reference to the $\mathrm{Ag}(111) \mathrm{SBZ}$ (green hexagon). 

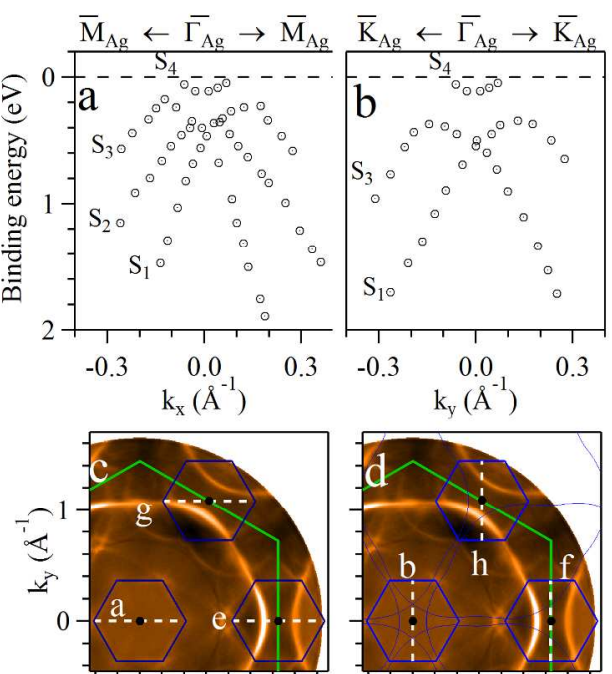

0

$\mathrm{k}_{\mathrm{x}}\left(\AA^{-1}\right)$ $\overline{\mathrm{M}}_{\mathrm{A}}$
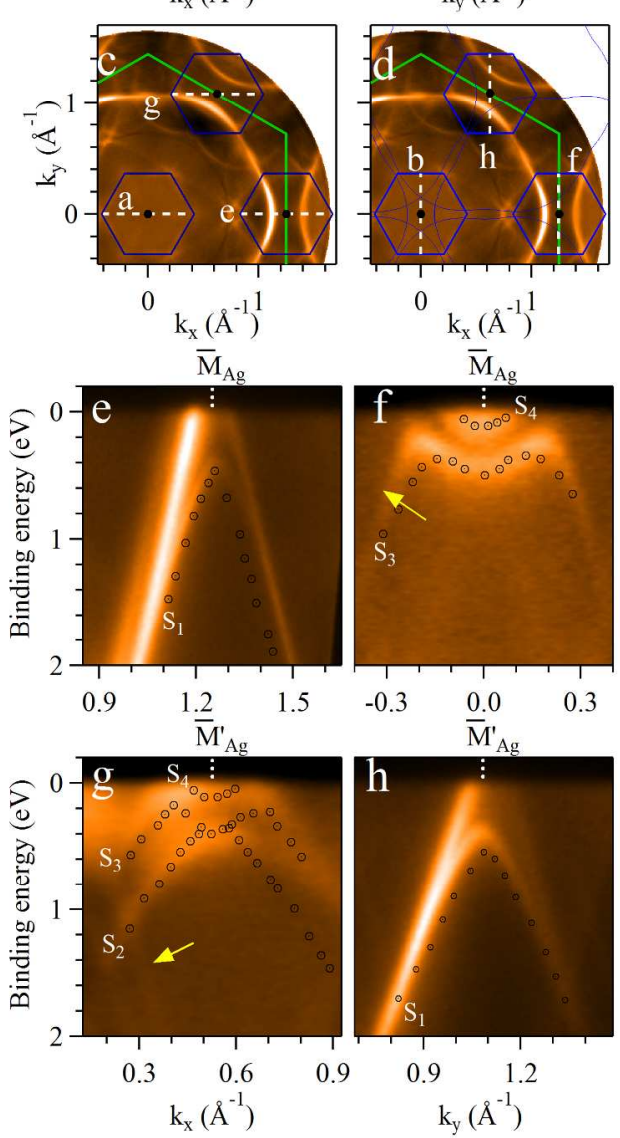

Figure 6. Top row: dispersion of the S1-S4 bands of the $(2 \sqrt{ } 3 \times 2 \sqrt{ } 3) \mathrm{R} 30^{\circ}$ system in the proximity of $\Gamma$ along the (a) $\Gamma \_A g-M \_A g$ and (b) $\Gamma \_A g-K \_A g$ directions, from the ref 25. (c) Location of the ARPES scans "e" (panel (e)) and " $g$ " (panel $(\mathrm{g})$ ) and equivalence with the line " $a$ " (blue and green hexagons indicate the SBZ of $(2 \sqrt{ } 3 \times 2 \sqrt{ } 3) R 30^{\circ}$ silicene and $\left.\mathrm{Ag}(111)\right)$. The constant energy cut in the background is a zoom of Fig. 3(i). (d) Location of the ARPES scans " $f$ " (panel (f)) and " $h$ " (panel $(h)$ ) and equivalence with the line " $b$ " (blue and green hexagons indicate the SBZ of $(2 \sqrt{ } 3 \times 2 \sqrt{ } 3) \mathrm{R} 30^{\circ}$ silicene and $\mathrm{Ag}(111)$. Thin blue lines indicate umklapp replicas of the constant energy contour of band $B$. The constant energy map in the background is a zoom of Fig. 3(i).

$112 \times 276 \mathrm{~mm}(300 \times 300 \mathrm{DPI})$ 


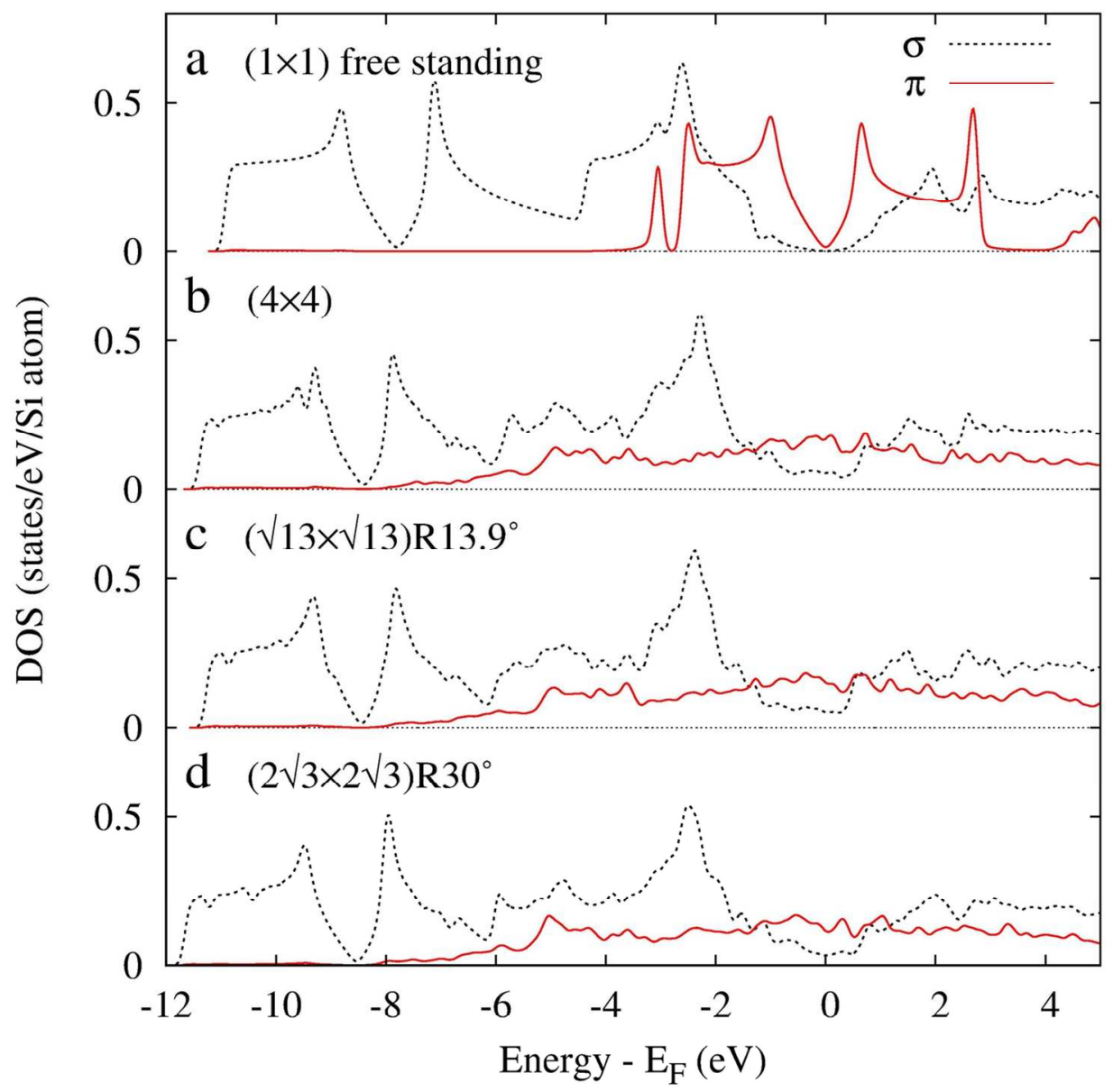

Figure 7. Si-projected DOS computed for (a) free-standing $(1 \times 1)$ low-buckled silicene and for (b) $(4 \times 4)$, (c) $(\sqrt{ } 13 \times \sqrt{ } 13) \mathrm{R} 13.9^{\circ}$ and $(\mathrm{d})(2 \sqrt{ } 3 \times 2 \sqrt{ } 3) \mathrm{R} 30^{\circ} \mathrm{Ag}(111)$-supported silicene layers. $\sigma(\mathrm{s}, \mathrm{px}$, and $\mathrm{py})$ and $\mathrm{n}(\mathrm{pz})$ components are indicated by dotted blue and continuous red lines, respectively.

$125 \times 124 \mathrm{~mm}(300 \times 300$ DPI $)$ 


\title{
Electronic States of Silicene Allotropes on $\mathrm{Ag}(111)$
}

\author{
Polina M. Sheverdyaeva, ${ }^{, \dagger}$ Sanjoy Kr. Mahatha, ${ }^{\dagger, t}$ Paolo Moras, ${ }^{\dagger}$ Luca Petaccia, ${ }^{\S}$ Guido \\ Fratesi, ${ }^{\perp}$ Giovanni Onida, ${ }^{\perp}$ and Carlo Carbone ${ }^{\dagger}$ \\ ${ }^{\dagger}$ Istituto di Struttura della Materia, Consiglio Nazionale delle Ricerche, SS 14, Km 163,5, I- \\ 34149 Trieste, Italy \\ ${ }^{\S}$ Elettra Sincrotrone Trieste, SS 14, Km 163,5, I-34149 Trieste, Italy \\ ${ }^{\perp}$ Università degli Studi di Milano, Via Celoria, 16, I-20133 Milano, Italy \\ *polina.sheverdyaeva@trieste.ism.cnr.it
}

\begin{abstract}
Silicene, a honeycomb lattice of silicon, presents a particular case of allotropism on $\operatorname{Ag}(111)$. Silicene forms multiple structures with alike in-plane geometry but different out-of-plane atomic buckling and registry to the substrate. Angle-resolved photoemission and first-principles calculations show that these silicene structures, with $(4 \times 4),(\sqrt{13} \times \sqrt{13}) \mathrm{R} 13.9^{\circ}$ and $(2 \sqrt{3} \times 2 \sqrt{ } 3) \mathrm{R} 30^{\circ}$ lattice periodicity, display similar electronic bands despite the structural
\end{abstract}


2

differences. In all cases the interaction with the substrate modifies the electronic states, which result to significantly differ from the ones of free-standing silicene. Complex photoemission patterns arise from surface umklapp processes, varying according to the periodicity of the silicene allotropes.

KEYWORDS: silicene, low energy electron diffraction (LEED), angle-resolved photoemission spectroscopy (ARPES), density functional theory (DFT). 
Silicene, a two-dimensional (2D) honeycomb lattice of silicon, is theoretically predicted to be stable as a free-standing monolayer in a low-buckled structure. ${ }^{1}$ Like graphene, it displays Dirac cones at the $\mathrm{K}_{\mathrm{Si}}$ points near the Fermi level $\left(\mathrm{E}_{\mathrm{F}}\right)$ arising from the nearly linear dispersion of $\pi$ symmetry electronic states. ${ }^{1,2}$ Due to the larger spin-orbit coupling of Si compared to C, freestanding silicene would exhibit interesting spintronic properties, including a non-trivial topological character, ${ }^{3}$ quantum spin Hall effect, ${ }^{4}$ and electrically tunable band gaps. ${ }^{5}$

Although free-standing silicene could not be produced until now, experimental studies reported the epitaxial synthesis of silicon monolayer, organized in a honeycomb lattice, on different substrates, such as $\operatorname{Ag}(111),{ }^{6-14} \mathrm{ZrB}_{2}(0001),{ }^{15,16}$ and $\operatorname{Ir}(111) .{ }^{17}$ These Si honeycomb structures are commonly referred to as silicene, although the free standing silicene properties are generally modified by the substrate interaction.

Silicene on $\operatorname{Ag}(111)$, which is the best studied case, displays the particular property of forming a manifold of allotropic structures, depending on the growth temperature and Si coverage. ${ }^{6,11,13}$ These honeycomb structures have a similar in-plane atomic geometry, with inter-atomic distances close to those expected for free-standing silicene. They differ with respect to the registry relations to the substrate, out of plane atomic buckling, and surface periodicity. Scanning tunneling microscopy (STM) and low-energy electron diffraction (LEED) report silicene structures with $(4 \times 4),(\sqrt{ } 13 \times \sqrt{ } 13) \mathrm{R} 13.9^{\circ}$ and $(2 \sqrt{3} \times 2 \sqrt{ } 3) \mathrm{R} 30^{\circ}$ periodicity compared to the $\mathrm{Ag}$ unit cell, besides minor phases observed in the early growth stages. ${ }^{6,13}$ Fig. 1 schematically represents the structural models (top row, derived from ref 18) and surface Brillouin zones (SBZ, bottom row) of the $(4 \times 4),(\sqrt{13} \times \sqrt{ } 13) \mathrm{R} 13.9^{\circ}$ and $(2 \sqrt{3} \times 2 \sqrt{3}) \mathrm{R} 30^{\circ}$ silicene allotropes. The unit cells present different rotational angles with respect to the substrate (angle $\alpha$ between the $\mathrm{Si}[110]$ and $\operatorname{Ag}[110]$ directions). Total-energy electronic structure calculations derive structural geometries in 
good agreement with the STM experimental observations. ${ }^{18}$ The theoretical results also show that the silicene allotropes are nearly degenerate, with cohesive energy between Si and Ag in the range of a weak covalent bonding, accounting thus for their coexistence under most or all growth conditions. $^{13,18}$

Figure 1. Top row: structural model of the silicene allotropes on $\operatorname{Ag}(111)$ (derived from ref 18). Grey circles represent Ag atoms. Blue circles represent Si atoms located at different height (circle size and color brightness increase with increasing the distance from the Ag substrate). Blue rhombi define the unit cells of the silicene superstructures. The angle $\alpha$ between the $\operatorname{Si}[110]$ and $\mathrm{Ag}[110]$ directions is characteristic of each phase (a) $(4 \times 4), \alpha=0^{\circ}$; (b) $(\sqrt{ } 13 \times \sqrt{ } 13) \mathrm{R} 13.9^{\circ}$, $\alpha=5.2^{\circ}$; (c) $(2 \sqrt{ } 3 \times 2 \sqrt{ } 3) \mathrm{R} 30^{\circ}, \alpha=10^{\circ}$. Bottom row: reciprocal space of the silicene allotropes on $\operatorname{Ag}(111)$. Thin blue hexagons indicate the SBZ of the silicene reconstruction. Thick blue and green hexagons represent the $(1 \times 1) \mathrm{SBZ}$ of silicene and $\operatorname{Ag}(111)$, respectively. Dashed hexagons in (b) and (c) are used to highlight the presence of a second rotational domain.

A key question regarding the fundamental and applicative properties of silicene is whether the presence of Dirac fermions is preserved on a supporting substrate. Studies aiming to establish to what extent silicene on $\operatorname{Ag}(111)$ retains the electronic structure of free standing silicene were mainly focused on the $(4 \times 4)$ structure. Early angle-resolved photoemission spectroscopy (ARPES) data have been interpreted as providing evidence for linearly dispersing bands close to $\mathrm{E}_{\mathrm{F}}{ }^{10,19}$ like the Dirac cones in graphene. Experimental ${ }^{12,14,20}$ and theoretical ${ }^{12,14,21-23}$ investigations have more recently demonstrated that this assignment is not correct, since the spectral features of the $(4 \times 4)$ structure originally interpreted as silicene bands derive from bulk 
and interface Ag states. These studies show that the $\pi$-symmetry states become delocalized due to the hybridization with the substrates, lose the $2 \mathrm{D}$ honeycomb character, and do not form Dirac cones. The $\sigma$-bands turn out to be less interacting and moderately affected by the substrate. ${ }^{14,24}$

The effect of the electronic interaction with the substrate for the $(\sqrt{ } 13 \times \sqrt{ } 13) \mathrm{R} 13.9^{\circ}$ and $(2 \sqrt{ } 3 \times 2 \sqrt{ } 3) \mathrm{R} 30^{\circ}$ structures is largely unexplored. While the $(\sqrt{ } 13 \times \sqrt{ } 13) \mathrm{R} 13.9^{\circ}$ structure has not been examined yet by ARPES, a recent study addressed the $(2 \sqrt{ } 3 \times 2 \sqrt{ } 3) \mathrm{R} 30^{\circ}$ structure, ${ }^{25}$ but the absence or presence of the Dirac cone were not established. Contrary to the $(4 \times 4)$ structure, ARPES results on the $(2 \sqrt{3} \times 2 \sqrt{3}) \mathrm{R} 30^{\circ}$ phase find a manifold of $\sigma$ bands shifted much closer to $\mathrm{E}_{\mathrm{F}}$ than in free-standing silicene, besides the formation of an interface state with energy similar to the one observed on the $(4 \times 4)$ structure. $^{25}$ These observations raise therefore the question whether and to what extent silicene allotropes on $\operatorname{Ag}(111)$ present dissimilar electronic properties.

Here we compare the electronic structure of $(4 \times 4),(\sqrt{13} \times \sqrt{13}) \mathrm{R} 13.9^{\circ}$ and $(2 \sqrt{ } 3 \times 2 \sqrt{ } 3) \mathrm{R} 30^{\circ}$ silicene monolayers on $\operatorname{Ag}(111)$ by means of ARPES and first-principles calculations. We find that all phases display similar Ag-derived interface states and $\sigma$ bands weakly perturbed by the substrate interaction. Intense spectral features, including those previously attributed to $\sigma$ band emission from the $(2 \sqrt{3} \times 2 \sqrt{3}) \mathrm{R} 30^{\circ}$ structure, are found to originate from umklapp replicas of the Ag interface state and Ag $s p$-bulk bands. All the examined silicene allotropes do not display the characteristic Dirac cones of free-standing silicene, proving that the $\pi$ bands are strongly modified by the interaction with the substrate bands independently of the structural detail of the allotrope.

\section{RESULTS AND DISCUSSION}


Growth of Silicene Allotropes on Ag(111). Fig. 2(a) shows the LEED pattern of the $(4 \times 4) /(\sqrt{ } 13 \times \sqrt{ } 13) \mathrm{R} 13.9^{\circ}$ system. The $(0,0)$ specular reflection and the $\operatorname{Ag}(1,0)$ spot are indicated for reference. Arrows mark the first order spots of the $(4 \times 4)$ domains (central spot) and of the twin $(\sqrt{ } 13 \times \sqrt{ } 13) \mathrm{R} 13.9^{\circ}$ domains with respect to the $(0,0)$ reflection. The same set of spots is replicated on the left hand side of the figure (enclosed within the oval area) and refers to the $\operatorname{Ag}(-$ 1,0) spot (not present in the LEED pattern). This assignment is illustrated in Fig. 2(b) by circles (dashed lines) that link silicene diffraction spots (blue dots) to specific Ag diffraction spots $\left(\bar{\Gamma}_{\mathrm{Ag}}\right.$ points). Fig. 2(a) shows also the weak first order spots of the twin $(\sqrt{ } 13 \times \sqrt{ } 13) \mathrm{R} 13.9^{\circ} b$ domains (rectangle area), a low-ordered silicene phase which often coexists with well-ordered $(\sqrt{ } 13 \times \sqrt{ } 13) \mathrm{R} 13.9^{\circ}$ and $(4 \times 4)$ domains. ${ }^{13}$ Its electronic properties are briefly addressed in the Supporting Information.

Figure 2. (a) LEED pattern at $31 \mathrm{eV}$ of the $(4 \times 4) /(\sqrt{ } 13 \times \sqrt{ } 13) \mathrm{R} 13.9^{\circ}$ system. Some first-order diffraction spots of Ag and silicene are indicated by arrows. The oval area encloses first-order diffraction spots of silicene back-scattered from the second SBZ of Ag. The blurred spots in the squared area are associated to the low-ordered $(\sqrt{ } 13 \times \sqrt{ } 13) \mathrm{R} 13.9^{\circ} \mathrm{b}$ phase. (b) Schematic diagram of the LEED pattern of panel (a) that shows the origin of the back-scattered silicene spots (blue dots) by dashed circular lines. (c) LEED pattern at $31 \mathrm{eV}$ of the $(2 \sqrt{3} \times 2 \sqrt{ } 3) \mathrm{R} 30^{\circ}$ system. (d) Same as panel (b) for the $(2 \sqrt{3} \times 2 \sqrt{ } 3) \mathrm{R} 30^{\circ}$ system.

The distribution of primary and back-scattered silicene spots in the LEED pattern of the $(2 \sqrt{3} \times 2 \sqrt{ } 3) \mathrm{R}^{\circ} 0^{\circ}$ system (Fig. 2(c)) can be described in a similar way (Fig. 2(d)). The $(2 \sqrt{3} \times 2 \sqrt{ } 3) \mathrm{R} 30^{\circ}$ silicene allotrope forms larger domains, compared to the $(4 \times 4)$ and 
$(\sqrt{13} \times \sqrt{ } 13) \mathrm{R} 13.9^{\circ}$ structures, with minor presence of additional phases, ${ }^{13}$ and higher long range ordering, which results in shaper LEED spots.

Absence of Dirac Cones and Umklapp Scattering. Fig. 3(a,f) displays ARPES data measured for the mixed $(4 \times 4) /(\sqrt{ } 13 \times \sqrt{ } 13) \mathrm{R} 13.9^{\circ}$ system and the $(2 \sqrt{ } 3 \times 2 \sqrt{ } 3) \mathrm{R} 30^{\circ}$ one along directions passing through $\mathrm{K}_{\mathrm{Si}}$, where free-standing silicene presents Dirac cones. Namely, the $\Gamma_{\mathrm{Si}}-\mathrm{K}_{\mathrm{Si}}$ directions of the $(\sqrt{ } 13 \times \sqrt{ } 13) \mathrm{R} 13.9^{\circ}$ and $(2 \sqrt{3} \times 2 \sqrt{ } 3) \mathrm{R} 30^{\circ}$ phases are considered as marked by blue dashed lines in panels (d) and (i), respectively (a similar analysis for the $(4 \times 4)$ phase was reported previously ${ }^{14}$ and is not repeated here). The main observation is the intense feature marked $\mathrm{B}$ therein, with almost linear dispersion near $\mathrm{E}_{\mathrm{F}}$. Comparison with corresponding data for the clean $\operatorname{Ag}(111)$ surface (Fig. 3(b,g)) shows that this feature is an Ag sp-bulk band, which remains unaltered in the spectra of the silicene structures. No additional spectral feature, that could be attributed to the $\pi$ bands, is observed with the growth of the silicene layers in the proximity of $\mathrm{K}_{\mathrm{Si}}$. Also, no $\pi$ derived silicene band is found at any point in reciprocal space which by symmetry corresponds to $K_{S i}$ in the $(\sqrt{ } 13 \times \sqrt{ } 13) R 13.9^{\circ}$ and $(2 \sqrt{ } 3 \times 2 \sqrt{ } 3) R 30^{\circ}$ supercells. This observation is in line with results reported on the $(4 \times 4)$ structure $^{14,20}$ and implies that silicene fully loses its most characteristic electronic properties also in the $(\sqrt{ } 13 \times \sqrt{ } 13) \mathrm{R} 13.9^{\circ}$ and $(2 \sqrt{ } 3 \times 2 \sqrt{ } 3) \mathrm{R} 30^{\circ}$ allotropes on $\operatorname{Ag}(111)$.

Figure 3. Top row: ARPES analysis (31 eV photon energy) and simulations for the $(4 \times 4) /(\sqrt{ } 13 \times \sqrt{ } 13) R 13.9^{\circ}$ system. (a) ARPES data measured along the line indicated in panel (d) near the $K_{\text {si }}$ point of the $(\sqrt{ } 13 \times \sqrt{ } 13) \mathrm{R} 13.9^{\circ}$ phase. The white arrow indicates the $B$ band. (b) Same as panel (a) for the clean $\operatorname{Ag}(111)$. (c) Simulation of the band dispersion of panel (a), 
where thick green and thin blue lines represent the original B band and its umklapp replicas according to the $(4 \times 4)$ periodicity. (d) Constant energy cut of the ARPES data at $1 \mathrm{eV}$ below $\mathrm{E}_{\mathrm{F}}$. (e) Simulation of the band pattern of panel (d). Thick green and thin blue lines represent the energy contour of the original B band and its umklapp replicas according to the $(4 \times 4)$ periodicity. Thick blue lines highlight the umklapp bands observed in the experiment. Similarly, thick red lines mark the features observed in the experiments that derive from umklapp replicas of the $\mathrm{B}$ band repeated according to the $(\sqrt{ } 13 \times \sqrt{ } 13) \mathrm{R} 13.9^{\circ}$ periodicity (see text for more details). Black arrows facilitate the comparison between the ARPES pattern and the simulation. Bottom row: same as the top row for the $(2 \sqrt{3} \times 2 \sqrt{ } 3) \mathrm{R} 30^{\circ}$ system. Thick blue lines in panel (h) indicate some of the umklapp replicas of the B band observed in panel (f).

Fig. 3(f) displays, in addition to the intense substrate states, some faint bands, indicated by yellow arrows. Weak features characterize also constant energy cuts of the ARPES data, while dominated by the rounded hexagonal-like contour of the B band, as reported in Fig. 3(d,i) for an energy of $1 \mathrm{eV}$ below $\mathrm{E}_{\mathrm{F}}$. To identify the origin of such spectral features we need to take into account the umklapp processes, in which the photoemitted electrons exchange with the reciprocal lattice an in-plane vector of the reconstruction-induced superstructure. ${ }^{26-31} \mathrm{We}$ determine the position of the B band in the $\left(k_{x}, k_{y}\right)$ plane (see the thick green line in the schemes of Fig. $3(\mathrm{e}, \mathrm{j}))$, and replicate it in the $\left(k_{x}, k_{y}\right)$ plane with the periodicity dictated by the silicene allotrope. These replicas are reported as thin blue lines in Fig. 3(e,j) for the $(4 \times 4)$ and $(2 \sqrt{ } 3 \times 2 \sqrt{ } 3) \mathrm{R} 30^{\circ}$ superlattices, respectively. Thick blue lines in Fig. 3(e,j) highlight the clearest correspondences between the experimental data and the simulation. In the case of the $(4 \times 4) /(\sqrt{ } 13 \times \sqrt{ } 13) \mathrm{R} 13.9^{\circ}$ system, additional replicas following the $(\sqrt{ } 13 \times \sqrt{ } 13) \mathrm{R} 13.9^{\circ}$ periodicity (thick red lines) are necessary to describe the position of weak features near the borders of the 
$\operatorname{Ag}(111)$ SBZ. The higher structural order of the $(2 \sqrt{3} \times 2 \sqrt{3}) \mathrm{R} 30^{\circ}$ silicene layer results in umklapp features of enhanced sharpness, and allows an easier comparison with the corresponding simulated pattern in Fig. 3(i,j) (black arrows).

By repeating the simulation of the photoemission signal on constant energy planes for all investigated binding energies $(0-3 \mathrm{eV})$ it is possible to derive the expected energy-momentum band dispersion in the presence of bulk umklapp scattering. This procedure yields the bands in Fig. 3(c) for the $(4 \times 4) /(\sqrt{13} \times \sqrt{13}) \mathrm{R} 13.9^{\circ}$ system, where no umklapp features are identified in the ARPES spectra of Fig. 3(a) along this specific line. If applied to the $(2 \sqrt{3} \times 2 \sqrt{3}) \mathrm{R} 30^{\circ}$ system, the same procedure gives the simulated bands of Fig. 3(h). Thick blue lines mark the position of the umklapp bands that can be observed in Fig. 3(f) (yellow arrows). Hence these spectral features do not represent emission from silicene states, but arise from photoemission of Ag states. These findings demonstrate that the umklapp scattering effects must be taken into consideration for the understanding of the ARPES data for silicene layers on $\operatorname{Ag}(111)$.

Interface State Formation. An interface state of $s p$-Ag character was found by ARPES in the $(4 \times 4)$ structure. ${ }^{14,20}$ A state with similar binding energy and dispersion was also observed on the $(2 \sqrt{ } 3 \times 2 \sqrt{ } 3) \mathrm{R}^{\circ} 0^{\circ}$ structure. $^{25}$ Fig. 4 shows a comparison between the ARPES data of the $(4 \times 4) /(\sqrt{ } 13 \times \sqrt{ } 13) \mathrm{R} 13.9^{\circ}$ and $(2 \sqrt{ } 3 \times 2 \sqrt{ } 3) \mathrm{R} 30^{\circ}$ systems near the $\overline{\mathrm{M}}_{\mathrm{Ag}}$ point (the scanned lines with reference to the $\operatorname{Ag}(111) \mathrm{SBZ}$ are shown in Fig. 4(e)). In both cases the interface state (I) has a maximum along the $\bar{\Gamma}_{\mathrm{Ag}}-\overline{\mathrm{M}}_{\mathrm{Ag}}$ axis (Fig. 4(a,c)) and a minimum along the perpendicular $\overline{\mathrm{K}}_{\mathrm{Ag}}-\overline{\mathrm{M}}_{\mathrm{Ag}}-\overline{\mathrm{K}}_{\mathrm{Ag}}$ direction (Fig. 4(b,d)) at approximately the same binding energy $(0.4 \mathrm{eV})$. These measurements demonstrate that the I state, also for the $(2 \sqrt{3} \times 2 \sqrt{3}) \mathrm{R} 30^{\circ}$, has a saddle-like dispersion in the vicinity of the $\bar{M}_{\mathrm{Ag}}$ point. Theoretical examination of the I state formed on the (4×4) structure shows that this state is largely of $\mathrm{Ag} s p$-derived character, lies in the $\operatorname{Ag}(111)$ 
bulk projected gap, and is localized mainly in the first two Ag layers under the silicene. ${ }^{14}$ It appears very likely that the state observed in the $(2 \sqrt{3} \times 2 \sqrt{3}) \mathrm{R} 30^{\circ}$ structure has the same origin and character. We notice that a similar state is also observed near the $\overline{\mathrm{M}}_{\mathrm{Ag}}$ point when Sn forms an ordered $(\sqrt{ } 3 \times \sqrt{ } 3) \mathrm{R} 30^{\circ}$ superstructure on $\operatorname{Ag}(111) .^{32}$ The features above the I states in Fig. 4(b,d) derive from the tail of the Ag bulk bands, which are located just above $\mathrm{E}_{\mathrm{F}}$, while other weaker structures marked by yellow arrows, arise from umklapp replicas of the B band, as shown above.

Figure 4. Top row: ARPES data $\left(31 \mathrm{eV}\right.$ photon energy) for the $(4 \times 4) /(\sqrt{13} \times \sqrt{ } 13) \mathrm{R} 13.9^{\circ}$ system measured in the proximity of the $\overline{\mathrm{M}}_{\mathrm{Ag}}$ point along the (a) $\bar{\Gamma}_{\mathrm{Ag}}-\overline{\mathrm{M}}_{\mathrm{Ag}}$ and (b) $\overline{\mathrm{K}}_{\mathrm{Ag}}-\overline{\mathrm{M}}_{\mathrm{Ag}}-\overline{\mathrm{K}}_{\mathrm{Ag}}$ directions. Line scans are shown in panel (e) with reference to the Ag SBZ. Yellow arrows indicate umklapp replicas of the $\mathrm{B}$ band. Bottom row: same as the top row for the $(2 \sqrt{ } 3 \times 2 \sqrt{ } 3) \mathrm{R} 30^{\circ}$ system.

б-bands. Fig. $5\left(\right.$ a) shows the electronic structure of the $(4 \times 4) /(\sqrt{ } 13 \times \sqrt{ } 13) \mathrm{R} 13.9^{\circ}$ system along the $\bar{\Gamma}_{\mathrm{Ag}}-\overline{\mathrm{M}}_{\mathrm{Ag}}$ direction, where B and I are the most intense bands. Half-way between $\bar{\Gamma}_{\mathrm{Ag}}$ and $\overline{\mathrm{M}}_{\mathrm{Ag}}\left(\Gamma_{4 \times 4}\right.$ at $\left.k_{x}=0.63 \AA^{-1}\right)$ there is a downward dispersive band with maximum at about $1.3 \mathrm{eV}$ binding energy (better seen in the zoom of Fig. 5(b)), which corresponds to a replica of the $\sigma$ band reported in ref 14 for the same system. This feature appears broader in the perpendicular direction, i.e. along $K_{4 \times 4}-\Gamma_{4 \times 4}-K_{4 \times 4}$, due to the coexistence of $\sigma$ states of the $(4 \times 4)$ and $(\sqrt{13} \times \sqrt{ } 13) \mathrm{R} 13.9^{\circ}$ domains with similar band properties (Fig. 5(c)). Fig. 5(d) shows the location of the scanned directions in the reciprocal space. It turns out that the observed $\sigma$ bands are located in correspondence to the first order diffraction spot of the $(4 \times 4)$ and $(\sqrt{ } 13 \times \sqrt{ } 13) \mathrm{R} 13.9^{\circ}$ 
phases back-scattered from the second zone of Ag near the $(0,0)$ reflection (Fig. 2(a,b)). This information is further confirmed by the dispersion of the $\sigma$ bands extracted at higher photon energies (see ref 14 and Supporting information).

Figure 5. Top row: $\sigma$ bands of the $(4 \times 4) /(\sqrt{ } 13 \times \sqrt{ } 13) R 13.9^{\circ}$ system. ARPES scans along the $(a, b)$ $\bar{\Gamma}_{\mathrm{Ag}}-\overline{\mathrm{M}}_{\mathrm{Ag}}$ and (c) $\mathrm{K}_{4 \times 4}-\Gamma_{4 \times 4}-\mathrm{K}_{4 \times 4}$ directions (photon energy $31 \mathrm{eV}$ ). Dashed lines highlight the $\sigma$ band dispersions. Yellow arrows in panel (a) indicate umklapp replicas of the B band. (d) Location of the symmetry points and SBZ (blue hexagons) of the $(4 \times 4)$ superstructure with reference to the $\operatorname{Ag}(111) \mathrm{SBZ}$ (green hexagon). Bottom row: $\sigma$ bands of the $(2 \sqrt{3} \times 2 \sqrt{ } 3) \mathrm{R} 30^{\circ}$ system. ARPES scans along the (e,f) $\bar{\Gamma}_{A g}-\bar{K}_{A g}$ and $(g) K_{2 \sqrt{ } 3}-\Gamma_{2 \sqrt{ } 3}-K_{2 \sqrt{ } 3}$ directions (photon energy $31 \mathrm{eV}$ ). Dashed lines highlight the $\sigma$ band dispersions. Arrows in panel (e) indicate umklapp replicas of the $B$ band (yellow) as well as the $S_{1}$ state (cyan). (f) Location of the symmetry points and SBZ (blue hexagons) of the $(2 \sqrt{3} \times 2 \sqrt{3}) \mathrm{R} 30^{\circ}$ superstructure with reference to the $\mathrm{Ag}(111) \mathrm{SBZ}$ (green hexagon). 
Considering the $(2 \sqrt{3} \times 2 \sqrt{ } 3) \mathrm{R} 30^{\circ}$ system, Fig. 5(e,f) reports ARPES data along the $\bar{\Gamma}_{\text {Ag }}-\overline{\mathrm{K}}_{\text {Ag }}$ direction, where a $\sigma$ band with maximum at about $1.3 \mathrm{eV}$ is seen at $\Gamma_{2 \sqrt{3}}\left(k_{y}=0.73 \AA^{-1}\right.$ in Fig. 5(h)). Again, the observed feature is found in correspondence with the first order diffraction spot of the $(2 \sqrt{3} \times 2 \sqrt{ } 3) \mathrm{R} 30^{\circ}$ phase back-scattered near the $(0,0)$ reflection (Fig. 2(c,d), see also Supporting Information). The coexistence of twin domains, rotated by $\pm 10^{\circ}$ with respect to the $\operatorname{Ag}[110]$ axis in the real space, manifests in the appearance of two downward dispersing bands along the $\mathrm{K}_{2 \sqrt{3}}-\Gamma_{2 \sqrt{3}}-\mathrm{K}_{2 \sqrt{ } 3}$ direction in Fig. 5(g). The dispersion of the $\sigma$ states in the $(2 \sqrt{ } 3 \times 2 \sqrt{ } 3) \mathrm{R} 30^{\circ}$ system is hence very similar to that of the $(4 \times 4) /(\sqrt{ } 13 \times \sqrt{ } 13) \mathrm{R} 13.9^{\circ}$ one.

In summary, for both the $(4 \times 4) /(\sqrt{ } 13 \times \sqrt{ } 13) \mathrm{R} 13.9^{\circ}$ and $(2 \sqrt{3} \times 2 \sqrt{ } 3) \mathrm{R} 30^{\circ}$ systems the top of the $\sigma$ bands is located at the center of the respective $\mathrm{SBZ}$ at about $1.3 \mathrm{eV}$ below $\mathrm{E}_{\mathrm{F}}$. Instead, the broad $\sigma$ bands of the low-ordered $(\sqrt{ } 13 \times \sqrt{ } 13) \mathrm{R} 13.9^{\circ} \mathrm{b}$ phase are found at deeper energies (see Supporting Information).

As a last point of this section we want to comment on the presence of the band labeled $\mathrm{S}_{1}$ in Fig. 5(e). This feature was observed by ARPES in ref 25 and interpreted, along with other three bands in the same energy-momentum region (labeled $\left.S_{2}-S_{4}\right)$, as $(2 \sqrt{ } 3 \times 2 \sqrt{ } 3) R 30^{\circ}$-related bands of $\sigma$ character. We hereby present evidences for revisiting such interpretation in terms of umklapp scattering effects in the ARPES data. Fig. 6(a,b) reports the experimental dispersion of the $\mathrm{S}_{1}-\mathrm{S}_{4}$ states near $\bar{\Gamma}_{\mathrm{Ag}}$ along the $\bar{\Gamma}_{\mathrm{Ag}}-\overline{\mathrm{M}}_{\mathrm{Ag}}$ and $\bar{\Gamma}_{\mathrm{Ag}}-\overline{\mathrm{K}}_{\mathrm{Ag}}$ directions extracted from ref 25. Fig. 6(c) presents the SBZ for the $(2 \sqrt{3} \times 2 \sqrt{ } 3) \mathrm{R} 30^{\circ}$ system (superimposed on the constant energy cut at 1.0 eV binding energy from Fig. 3(i)) showing that lines "e" and "g" crossing the $\bar{M}_{A g}$ and $\bar{M}_{A g}^{\prime}$ points back-fold onto line "a" near $\bar{\Gamma}$, due to the symmetry of the $(2 \sqrt{3} \times 2 \sqrt{ } 3) \mathrm{R} 30^{\circ}$ superstructure. ARPES scans demonstrate that along line "e" the dispersion of band I matches with state $\mathrm{S}_{1}$ (Fig. 6(e)), as well as that along line "g" the dispersion of bands B and I matches with states $\mathrm{S}_{2}-\mathrm{S}_{4}$ 
(Fig. 6(g)). Similarly, lines "f" and "h" back-fold onto line "b" (Fig. 6(d)). Again, the features observed in Fig. 6(f,h) derive from bands B and I and closely reproduce the dispersion of the $\mathrm{S}_{3}-$ $\mathrm{S}_{4}$ and $\mathrm{S}_{1}$ states, respectively. Finally, umklapp replicas of the constant energy contour of band B, reported as thin blue lines in Fig. 6(d), give rise to a star-like pattern in the proximity of the $\bar{\Gamma}_{\text {Ag }}$ point, analogous to the one observed in the ARPES data and ascribed to the $\mathrm{S}_{1}$ and $\mathrm{S}_{2}$ states in ref 25. These results indicate that all the states marked as $S_{1}-S_{4}$ in ref 25 derive from umklapp scattering of Ag-related bands, either the B or the I band, rather than from silicene bands of $\sigma$ character.

Figure 6. Top row: dispersion of the $S_{1}-S_{4}$ bands of the $(2 \sqrt{3} \times 2 \sqrt{3}) R 30^{\circ}$ system in the proximity of $\bar{\Gamma}$ along the (a) $\bar{\Gamma}_{\mathrm{Ag}}-\overline{\mathrm{M}}_{\mathrm{Ag}}$ and (b) $\bar{\Gamma}_{\mathrm{Ag}}-\overline{\mathrm{K}}_{\mathrm{Ag}}$ directions, from the ref 25 . (c) Location of the ARPES scans "e" (panel (e)) and "g" (panel (g)) and equivalence with the line "a" (blue and green hexagons indicate the SBZ of $(2 \sqrt{3} \times 2 \sqrt{ } 3) \mathrm{R} 30^{\circ}$ silicene and $\left.\mathrm{Ag}(111)\right)$. The constant energy cut in the background is a zoom of Fig. 3(i). (d) Location of the ARPES scans " $\mathrm{f}$ " (panel (f)) and "h" (panel (h)) and equivalence with the line "b" (blue and green hexagons indicate the SBZ of $(2 \sqrt{3} \times 2 \sqrt{ } 3) \mathrm{R} 30^{\circ}$ silicene and $\operatorname{Ag}(111)$. Thin blue lines indicate umklapp replicas of the constant energy contour of band B. The constant energy map in the background is a zoom of Fig. 3(i).

Band Structure Calculations. As pointed out by theoretical and experimental studies, the symmetry and spatial extension of the silicene $p_{z}$ states, which form the $\pi$ bands, allow them to strongly hybridize with the $s p$-states of the $\operatorname{Ag}(111)$ substrate. ${ }^{14,21-23}$ The hybridization strength of the $p_{z}$ states with the $s p$-Ag band is expected to be similar and strong for all silicene allotropes, being in the range of a weak covalent bonding $(0.65-0.71 \mathrm{eV} /$ atom $),{ }^{18}$ although details may vary 
for the different phases. We briefly review here the computed electronic structure of silicene monolayers on $\operatorname{Ag}(111)$ for a consistent comparison with the experimentally investigated phases.

Firstly, we separate the effects of the structural deformations of silicene from the interaction with the substrate. Free-standing silicene monolayers with $(4 \times 4),(\sqrt{ } 13 \times \sqrt{ } 13) \mathrm{R} 13.9^{\circ}$ and $(2 \sqrt{ } 3 \times 2 \sqrt{ } 3) \mathrm{R} 30^{\circ}$ structures display significantly different band structures with respect to lowbuckled silicene. In the $(4 \times 4)$ phase the $K_{\mathrm{Si}}$ point is back-folded into $\bar{\Gamma}$, where a $0.3 \mathrm{eV}$ band gap opens. $^{21,33,34}$ Conversely, the free-standing $(\sqrt{ } 13 \times \sqrt{ } 13) \mathrm{R} 13.9^{\circ}$ and $(2 \sqrt{3} \times 2 \sqrt{ } 3) \mathrm{R} 30^{\circ}$ phases show linearly dispersing bands and no energy gap opening at the $\mathrm{K}$ point of the SBZ, where the $\mathrm{K}_{\mathrm{Si}}$ point folds, as shown in Fig. $1 .^{33}$

Figure 7. Si-projected DOS computed for (a) free-standing $(1 \times 1)$ low-buckled silicene and for (b) $(4 \times 4)$, (c) $(\sqrt{ } 13 \times \sqrt{ } 13) \mathrm{R} 13.9^{\circ}$ and (d) $(2 \sqrt{ } 3 \times 2 \sqrt{ } 3) \mathrm{R} 30^{\circ} \mathrm{Ag}(111)$-supported silicene layers. $\sigma(s$, $p_{x}$, and $\left.p_{y}\right)$ and $\pi\left(p_{z}\right)$ components are indicated by dotted blue and continuous red lines, respectively.

When Ag is included in the calculations the electronic properties of the silicene layers change dramatically with respect to the free-standing cases. In line with previous findings, ${ }^{14,21}$ the $\pi$ bands of $(4 \times 4)$ silicene delocalize into the $\mathrm{Ag}(111)$ substrate and have no mass-less character nor Dirac cone dispersion. The DOS projected onto the silicene layer (Fig. 7(b)) displays a metalliclike behavior for the silicene $\pi$ states, which are featureless and approximately constant around $\mathrm{E}_{\mathrm{F}}$. Due to the hybridization with $\mathrm{Ag}$ and the non-planar arrangement of the Si atoms which enhances $\pi-\sigma$ hybridization, also $\sigma$ states give rise to a small contribution to the DOS at $\mathrm{E}_{\mathrm{F}}$. More prominent $\sigma$-related features, located away from $\mathrm{E}_{\mathrm{F}}$, show clear analogies with those of the unsupported and low-buckled $(1 \times 1)$ silicene $($ Fig. $7(\mathrm{a}))$. 
Despite stripped-off silicene layers exhibit strong dependence on the structural parameters, the Ag-supported $(4 \times 4)($ Fig. $7(b)),(\sqrt{ } 13 \times \sqrt{ } 13) R 13.9^{\circ}$ (Fig. $7($ c) $)$, and $(2 \sqrt{ } 3 \times 2 \sqrt{ } 3)$ R30 $0^{\circ}$ (Fig. 7(d)) phases display very similar electronic structure for all energies (see also Supporting Information). In agreement with previous studies, ${ }^{21}$ we find that silicene becomes slightly negatively doped. Estimated by Löwdin population analysis, ${ }^{35}$ the electron transfer shows minimal dependence on the specific silicene structure and amounts to $0.23,0.21$, and 0.27 electrons per unit cell in the $(4 \times 4),(\sqrt{ } 13 \times \sqrt{ } 13) \mathrm{R} 13.9^{\circ}$, and $(2 \sqrt{ } 3 \times 2 \sqrt{ } 3) \mathrm{R} 30^{\circ}$ phase, respectively.

These results confirm that the substrate hybridization has much stronger effects on the electronic states of the silicene layers than the specific in-plane orientation and buckling of the silicon atoms. In particular, calculations support the absence of $\pi$-like Dirac cones near $\mathrm{E}_{\mathrm{F}}$ and the formation of $\sigma$-derived electronic states with very similar properties for all silicene phases, in agreement with the ARPES analysis.

\section{CONCLUSIONS}

We compared the electronic structure of different silicene allotropes grown on $\mathrm{Ag}(111)$ by ARPES and first-principles calculations. The $(4 \times 4),(\sqrt{13} \times \sqrt{ } 13) \mathrm{R} 13.9^{\circ}$ and $(2 \sqrt{ } 3 \times 2 \sqrt{ } 3) \mathrm{R} 30^{\circ}$ systems display very similar silicene bands of $\sigma$ character and the formation of Ag-derived interface states with saddle-like band dispersion. The identification of umklapp replicas in the ARPES data of the $(2 \sqrt{3} \times 2 \sqrt{3}) \mathrm{R} 30^{\circ}$ phase allows a reinterpretation of the features previously ascribed to silicene $\sigma$ bands. All the examined silicene allotropic structures do not show Dirac cones at $\mathrm{E}_{\mathrm{F}}$, that are instead characteristic of free-standing silicene. The absence of $\pi$-like Dirac cones finds explanation in the strength of the hybridization between silicene and Ag states, 
which also overwhelms the electronic effects of specific structural details of the different silicene allotropes.

\section{METHODS}

Sample preparation and LEED. The clean Ag(111) surface was prepared by repeated cycles of $\mathrm{Ar}^{+}$sputtering and annealing at $550^{\circ} \mathrm{C}$. The $\operatorname{Ag}(111)$ surface quality and cleanliness was confirmed by sharp LEED spots and intense L-gap Shockley surface state. Silicon was deposited by resistive heating of a Si wafer at a rate of 0.01-0.02 ML/min on a clean $\mathrm{Ag}(111)$ surface. The substrate was maintained at $\sim 240^{\circ} \mathrm{C}$ to produce a $(4 \times 4) /(\sqrt{ } 13 \times \sqrt{ } 13) \mathrm{R} 13.9^{\circ}$ monolayer and at $\sim 270^{\circ} \mathrm{C}$ to produce the prevalent $(2 \sqrt{3} \times 2 \sqrt{3}) \mathrm{R} 30^{\circ}$ monolayer. ${ }^{13}$ Previous studies show that the (4×4) phase is always found to be mixed with $(\sqrt{ } 13 \times \sqrt{ } 13) \mathrm{R} 13.9^{\circ}$ and other phases, ${ }^{6,8,13}$ to an extent that depends on the deposition temperature and coverage. Although silicene structure may appear to form a pure $(4 \times 4)$ in LEED for certain electron energy, ${ }^{7,10}$ LEED I-V spot analysis shows that it actually coexists with other structures. ${ }^{13}$ The proportion of $(4 \times 4)$ and $(\sqrt{13} \times \sqrt{13}) \mathrm{R} 13.9^{\circ}$ structures in the system we examine is of approximately $2: 1$. Both $(\sqrt{13} \times \sqrt{13}) \mathrm{R} 13.9^{\circ}$ and $(2 \sqrt{ } 3 \times 2 \sqrt{ } 3) \mathrm{R} 30^{\circ}$ phases present two equivalent mirror domains with respect to the $\operatorname{Ag}[110]$ direction.

ARPES. The ARPES experiments were performed at room temperature on the VUVPhotoemission and BaDElPh beamlines (Elettra, Italy) using the same experimental geometry in both cases. Linearly polarized light illuminated the sample at approximately $45^{\circ}$ with respect to the surface normal. The sample was rotated azimuthally. The analyzer slit was set parallel to the scattering plane of the experiment. 
First-principles calculations. We performed first-principles calculations in the framework of density functional theory (DFT) with the local density approximation (LDA) ${ }^{36}$ to the exchange and correlation functional. Computational parameters and optimized coordinates for the $(4 \times 4)$, $(\sqrt{ } 13 \times \sqrt{ } 13) \mathrm{R} 13.9^{\circ}$, and $(2 \sqrt{ } 3 \times 2 \sqrt{ } 3) \mathrm{R} 30^{\circ}$ silicene phases are taken from ref 34 . Namely, the system is modeled in the periodically repeated slab approach, where five-layer Ag slabs are taken as a model of the $\operatorname{Ag}(111)$ substrate with silicene adsorbed on one side only. The vacuum separation between adjacent slabs amounts to at least $17 \AA$. We used the Quantum-ESPRESSO package, ${ }^{37}$ which implements DFT calculations with atomic pseudopotentials and plane wave basis sets, to compute the electronic structure of the system with a kinetic energy cutoff of 32 Ry. The densities of states (DOS) have been evaluated by sampling the SBZ with a $12 \times 12$ mesh for $(4 \times 4)$ silicene and $16 \times 16$ for the other cases. The projection on the $\sigma\left(s, p_{x}\right.$, and $\left.p_{y}\right)$ and $\pi\left(p_{z}\right)$ states is performed by Löwdin population analysis ${ }^{35}$ and has been broadened by convolution with a Gaussian smearing of $0.1 \mathrm{eV}$ for plotting. 


\section{AUTHOR INFORMATION}

\section{Corresponding Author}

* (P.M.S.) E-mail: polina.sheverdyaeva@trieste.ism.cnr.it.

\section{Present Addresses}

"Present address: Aarhus University, Ny Munkegade 120, DK-8000 Aarhus C, Denmark

\section{Notes}

The authors declare no competing financial interest.

\section{ACKNOWLEDGMENT}

We acknowledge the "Progetto Premiale Materiali e Dispositivi Magnetici e Superconduttivi per Sensoristica e ICT” of the Italian Ministry of Education, University and Research (MIUR)

Supporting Information Available: ARPES data taken at 130 and $135 \mathrm{eV}$ photon energies; DFT calculations for the band dispersion and density of states of the silicene allotropes (PDF). This material is available free of charge via the Internet at http://pubs.acs.org.

\section{References}

(1) Cahangirov, S.; Topsakal, M.; Aktürk, V.; Şahin, H.; Ciraci, S. Two- And OneDimensional Honeycomb Structures of Silicon and Germanium. Phys. Rev. Lett. 2009, 102, 236804.

(2) Lebègue, S.; Eriksson, O.; Electronic Structure of Two-Dimensional Crystals from $A b$ Initio Theory. Phys. Rev. B 2009, 79, 115409. 
(3) Ezawa, M. A Topological Insulator and Helical Zero Mode in Silicene under an Inhomogeneous Electric Field. New J. Phys. 2012, 14, 033003.

(4) Liu, C.-C.; Feng, W.; Yao, Y. Quantum Spin Hall Effect in Silicene and Two-Dimensional Germanium. Phys. Rev. Lett. 2011, 107, 076802.

(5) Drummond, N. D.; Zólyomi, V.; Fal'ko, V. I. Electrically Tunable Band Gap in Silicene. Phys. Rev. B 2012, 85, 075423.

(6) Jamgotchian, H.; Colignon, Y.; Hamzaoui, N.; Ealet, B.; Hoarau, J. Y.; Aufray, B.; Biberian J. P. Growth of Silicene Layers on $\operatorname{Ag}(111)$ : Unexpected Effect of the Substrate Temperature. J. Phys.: Condens. Matter 2012, 24, 172001.

(7) Lin, C. L.; Arafune, R.; Kawahara, K.; Tsukahara, N.; Minamitani, E.; Kim, Y.; Takagi, N.; Kawai, M. Structure of Silicene Grown on Ag(111). Appl. Phys. Express 2012, 5, 045802.

(8) Chiappe, D.; Grazianetti, C.; Tallarida, G.; Fanciulli, M.; Molle, A. Local Electronic Properties of Corrugated Silicene Phases. Adv. Mater. 2012, 24, 5088.

(9) Feng, B.; Ding, Z. J.; Meng, S.; Yao, Y. G.; He, X. Y.; Cheng, P.; Chen, L.; Wu, K. H. Evidence of Silicene in Honeycomb Structures of Silicon on $\operatorname{Ag}(111)$. Nano Lett. 2012, 12, 3507.

(10) Vogt, P.; De Padova, P.; Quaresima, C.; Avila, J.; Frantzeskakis, E.; Asensio, M. C.; Resta, A.; Ealet, B.; Le Lay, G. Silicene: Compelling Experimental Evidence for Graphenelike Two-Dimensional Silicon. Phys. Rev. Lett. 2012, 108, 155501.

(11) Arafune, R.; Lin, C.; Kawahara, K.; Tsukahara, N.; Minamitani, E.; Kim, Y.; Takagi, N.; Kawai, M. Structural Transition of Silicene on Ag(111). Surf. Sci. 2013, 608, 297. 
(12) Lin, C. L.; Arafune, R.; Kawahara, K.; Kanno, M.; Tsukahara, N.; Minamitani, E.; Kim, Y.; Kawai, M.; Takagi, N. Substrate-Induced Symmetry Breaking in Silicene. Phys. Rev. Lett. 2013, 110, 076801 .

(13) Moras, P.; Mentes, T. O.; Sheverdyaeva, P. M.; Locatelli A.; Carbone, C. Coexistence of Multiple Silicene Phases in Silicon Grown on Ag(1 1 1). J. Phys.: Condens. Matter 2014, 26, 185001.

(14) Mahatha, S. K.; Moras, P.; Bellini, V.; Sheverdyaeva, P. M.; Struzzi, C.; Petaccia, L.; Carbone, C. Silicene on Ag(111): a Honeycomb Lattice without Dirac Bands. Phys. Rev. B 2014, 89, 201416(R).

(15) Fleurence, A.; Friedlein, R.; Ozaki, T.; Kawai, H.; Wang, Y.; Yamada-Takamura, Y. Experimental Evidence for Epitaxial Silicene on Diboride Thin Films. Phys. Rev. Lett. 2012, $108,245501$.

(16) Friedlein R., Fleurence A., Sadowski, Yamada-Takamura Y. Tuning of Silicene-Substrate Interactions with Potassium Adsorption. Appl. Phys. Lett. 2013, 102, 221603.

(17) Meng, L.; Wang, Y.; Zhang, L.; Du, S.; Wu, R.; Li, L.; Zhang, Y.; Li, G.; Zhou, H.; Hofer, W. A.; Gao, H.-J. Buckled Silicene Formation on $\operatorname{Ir}(111)$. Nano Lett. 2013, 13, 685.

(18) Guo, Z.-X.; Furuya, S.; Iwata, J.; Oshiyama, A. Absence and Presence of Dirac Electrons in Silicene on Substrates. Phys. Rev. B 2013, 87, 235435.

(19) Avila, J.; De Padova, P.; Cho, S.; Colambo, I.; Lorcy, S.; Quaresima, C.; Vogt, P.; Resta, A.; Le Lay, G.; Asensio, M. C. Presence of Gapped Silicene-Derived Band in the Prototypical (3×3) Silicene Phase on Silver (111) Surfaces. J. Phys.: Condens. Matter 2013, 25, 262001. 
(20) Tsoutsou, D.; Xenogiannopoulou, E.; Golias, E.; Tsipas, P.; Dimoulas, A. Evidence for Hybrid Surface Metallic Band in (4×4) Silicene on $\operatorname{Ag}(111)$. Appl. Phys. Lett. 2013, 103, 231604.

(21) Cahangirov, S.; Audiffred, M.; Tang, P.; Iacomino, A.; Duan, W.; Merino, G.; Rubio, A. Electronic Structure of Silicene on Ag (111): Strong Hybridization Effects. Phys. Rev. B 2013, $88,035432$.

(22) Chen, M. X.; Weinert, M. Revealing the Substrate Origin of the Linear Dispersion of Silicene/Ag(111). Nano Lett. 2014, 14, 5189.

(23) Ishida, H.; Hamamoto, Y.; Morikawa, Y.; Minamitani, E.; Arafune, R.; Takagi, N. Electronic Structure of the $4 \times 4$ Silicene Monolayer on Semi-Infinite Ag(111). New J. Phys. 2015, 17, 015013.

(24) Mahatha, S. K.; Moras, P.; Sheverdyaeva, P. M.; Bellini, V.; Mentes, T. O.; Locatelli, A.; Flammini, R.; Horn, K.; Carbone, C.; Absence of Dirac cones In Monolayer Silicene and Multilayer $\mathrm{Si}$ Films on $\mathrm{Ag}(111)$. J. Electron. Spectrosc. Relat. Phenom. DOI: 10.1016/j.elspec.2016.09.005

(25) Wang, W.; Olovsson, W.; Uhrberg, R. I. G. Experimental and Theoretical Determination of $\sigma$ Bands on (“ $2 \sqrt{3} \times 2 \sqrt{ } 3$ ”) Silicene Grown on $\operatorname{Ag}(111)$. Phys. Rev. B 2015, 92, 205427.

(26) Anderson, J.; Lapeyre, G. J. Chemisorption-Induced Surface Umklapp Processes in Angle-Resolved Synchrotron Photoemission from W(001). Phys. Rev. Lett. 1976, 36, 376.

(27) Westphal, D.; Goldmann, A. Adsorbate-Induced Umklapp Processes in Photoemission from $\mathrm{Cl}$ on Cu. Surf. Sci. 1983, 126, 253. 
(28) Mugarza, A.; Mascaraque, A.; Repain, V.; Rousset, S. ; Altmann, K. N.; Himpsel, F. J.; Koroteev, Yu. M.; Chulkov, E.V.; García de Abajo, F. J.; Ortega, J. E. Lateral Quantum Wells at Vicinal Au(111) Studied with Angle-Resolved Photoemission. Phys. Rev. B 2002, 66, 245419.

(29) Shikin, A. M.; Gorovikov, S. A.; Adamchuk, V. K.; Gudat, W.; Rader, O. Electronic Structure of Carbon Nanostripes. Phys. Rev. Lett. 2003, 90, 256803.

(30) Shikin, A. M.; Varykhalov, A.; Prudnikova, G. V.; Adamchuk, V. K.; Gudat, W.; Rader, O. Photoemission from Stepped W(110): Initial or Final State Effect? Phys. Rev. Lett. 2004, 93, 146802.

(31) Bengió, S.; Navarro, V.; González-Barrio, M. A.; Cortés, R.; Vobornik, I.; Michel, E. G.; Mascaraque, A. Electronic Structure of Reconstructed Au(100): Two-Dimensional and OneDimensional Surface States. Phys. Rev. B 2012, 86, 045426

(32) Osiecki, J. R.; Uhrberg, R. I. G. Alloying of Sn in the Surface Layer of Ag(111). Phys. Rev. B 2013, 87, 075441.

(33) Grazianetti, C.; Chiappe, D.; Cinquanta, E.; Tallarida, G.; Fanciulli, M.; Molle, A. Exploring the Morphological and Electronic Properties of Silicene Superstructures. Appl. Surf. Sci. 2014, 291, 109.

(34) Cinquanta, E.; Fratesi, G.; dal Conte, S.; Grazianetti, C.; Scotognella, F.; Stagira, S.; Vozzi, C.; Onida, G.; Molle, A. Optical Response and Ultrafast Carrier Dynamics of the Silicene-Silver Interface. Phys. Rev. B 2015, 92, 165427.

(35) Löwdin, P.-O. On the Non-Orthogonality Problem Connected with the Use of Atomic Wave Functions in the Theory of Molecules and Crystals. J. Chem. Phys. 1950, 18, 365. 
(36) Perdew, J. P.; Zunger, A. Self-Interaction Correction to Density-Functional Approximations for Many-Electron Systems. Phys. Rev. B 1981, 23, 5048.

(37) Giannozzi, P.; Baroni, S.; Bonini, N.; Calandra, M.; Car, R.; Cavazzoni, C.; Ceresoli, D.; Chiarotti, G. L.; Cococcioni, M.; Dabo, I.; Dal Corso, A.; Fabris, S.; Fratesi, G.; de Gironcoli, S.; Gebauer, R.; Gerstmann, U.; Gougoussis, C.; Kokalj, A.; Lazzeri, M.; Martin-Samos, L.; et al. QUANTUM ESPRESSO: a Modular and Open-Source Software Project for Quantum Simulations of Materials. J. Phys.: Condens. Matter 2009, 21, 395502. 


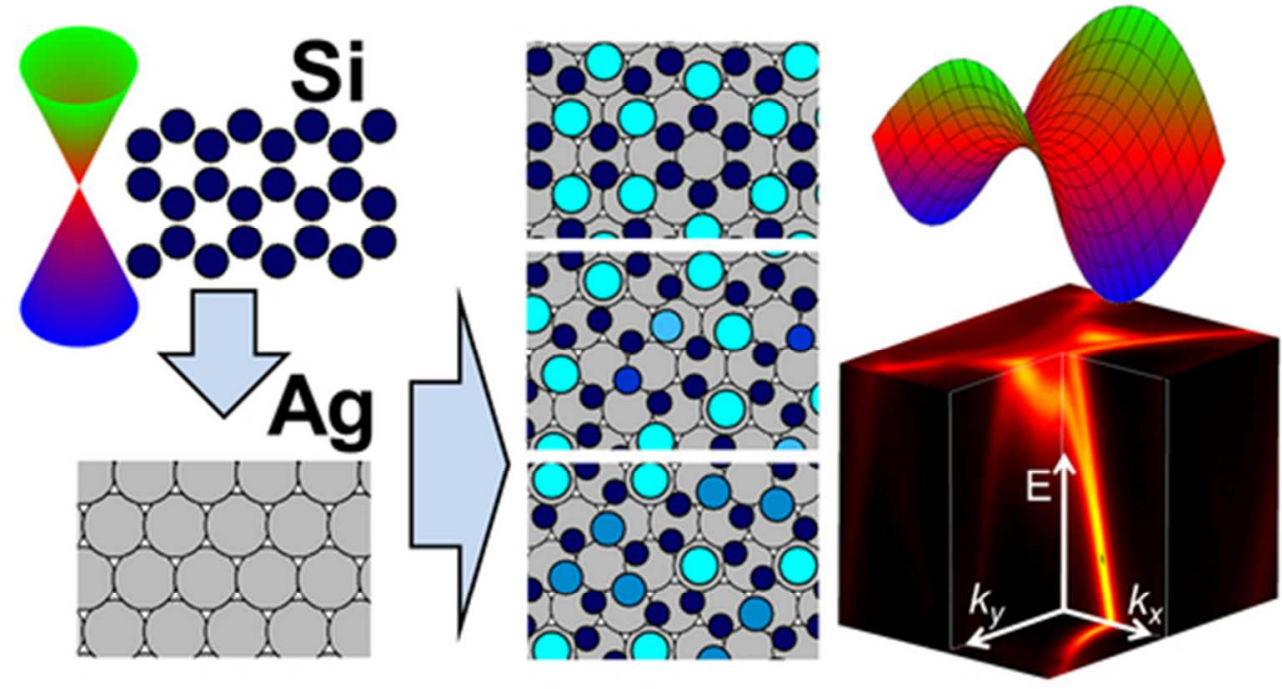

$44 \times 24 \mathrm{~mm}(300 \times 300$ DPI $)$ 